\title{
On market forces and human evolution
}

\author{
Gilles Saint-Paul*
}

May 31, 2006

\begin{abstract}
This paper studies how an institution such as markets affects the evolution of mankind. My key point is that the forces of natural selection are made weaker because trade allows people to specialize in those activities where they are strong, and to offset their weaknesses by purchasing adequate goods on the market. Absent trade, people must allocate their time among all the activities necessary for their fitness. A productivity advantage in any given dimension will increase survival probability, so that in the long run natural selection makes sure that population is entirely made of individuals with the strongest alleles at all loci. Under trade, there exist long-run equilibria where weaker individuals are able to achieve the same survival potential as the fittest, by specializing in activities where they are not at a disadvantage, and purchasing goods that are substitute for activities for which they are weak.
\end{abstract}

JEL classification: J1, J22

Keywords: Evolution, genotype, division of labor, specialization, geneculture coevolution, natural selection, trade, exchange, markets, time allocation

${ }^{*}$ Université de Toulouse I and CEPR. This paper has benefitted from discussions with Franjo Weissing, Omer Moav, Paul Seabright and Thierry Verdier, three anonymous reviewers from this journal, and from comments from seminar participants at the NBER summer institute, the Minerva conference, the Toulouse conference on biology and economics, the Maastricht workshop on human diversity and economic performance. 


\section{Introduction}

Basic Darwinian theory considers individuals competing for resources in order to achieve their goals of fitness and reproduction. Its more recent developments have considered cooperation and altruism as the outcome of selective forces at the gene level, which typically leave room for cooperation at a very small scale, within a family or tribe of genetically close individuals. Yet our species has developed institutions which allow cooperation on a much larger scale. ${ }^{1}$ Markets, in particular, allow people to specialize according to their comparative advantage and to purchase goods needed for fitness and survival from other participants. One may think that most present-day humans who make a living from specializing in narrow activities would have trouble surving if suddently left alone facing the forces of natural selection.

This paper studies how an institution such as markets affects the evolution of mankind. My key point is that the forces of natural selection are made weaker because trade allows people to specialize in those activities where they are strong, and to offset their weaknesses by purchasing adequate goods on the market. Absent trade, people must allocate their time among all the activities necessary for their fitness. A productivity advantage in any given dimension will increase survival probability, so that in the long run natural selection implies that population is entirely made of individuals with the best genes at all loci. Under trade, I define a long-run equilibrium as a situation where (i) markets clear at each period, and (ii) the distribution of genotypes in the population is stationary. I show that there exist longrun equilibria where weaker individuals are able to achieve the same survival potential as the strongest, by specializing in activities where they are not at a disadvantage, and purchasing goods that are substitute for activities for which they are 'weak'.

Hence, markets allow many genotypes that would be eliminated by natural selection to survive, thus building in greater genetic diversity in human

\footnotetext{
${ }^{1}$ Interesting surveys on interactions between the economic and biological spheres include Hirshleifer (1977), Robson (2001), and Seabright (2003), forthcoming.
} 
populations. As shown in the analysis below, this genetic diversity may be helpful in face of environmental shocks, implying that when such shocks prevail, a population which trades will grow faster than a population which does not, eventually eliminating it in statistical terms.

Note that the model is silent about how markets themselves evolve ${ }^{2}$. The literature on gene/culture coevolution is mostly concerned with the genetic basis for adoptions of cultural norms such as altruism (often relying on controversial group selection hypotheses). ${ }^{3}$ To my knowledge it has not provided a theory of how complex institutions such as markets evolve. The present paper only looks at causality in one dimension, taking institutions as given and studying their impact on the gene pool. The most closely related paper is Horan et al. (2002). They make a similar point that trade increases a population's fitness and partially offsets natural selection. They argue that trade may have played a role in the extinction of the Neanderthal and its replacement by homo sapiens. On the other hand, their model does not allow to study the evolution of the gene pool (as alleles and sexual reproduction are ignored), nor do they analyze the properties of a long-run equilibrium as defined here. A related literature (See Hammerstein (2003), and in particular Bowles and Hammerstein (2003)), studies the rise of markets and specialization in animal societies, but does not draw this paper's implications for the gene pool and the survival of alleles that would not survive absent arkets.

A potentially relevant critique - which applies to any causal link from culture to genes - is that it is not clear whether trade has been around for long enough to significantly affect evolution. It is often argued that evolution is very slow, and that our genes are essentially determined by the hunter-gatherer societies which prevailed hundreds of thousands of years ago. However, there are two quite different aspects of evolution. The first one is that mutations do not happen frequently, which explains why it takes

\footnotetext{
${ }^{2}$ Interestingly, Adam Smith saw our propensity to trade as a genetic property of our species. See Smith (1994).

${ }^{3}$ See Cavalli-Sforza and Feldman (1981); Lumsden and Wilson (1981); Gintis (2002), Boyd and Richerson (1985).
} 
hundreds of thousands of years for a feature like the human brain to develop. The second one is that an existing allele can replace another one quite rapidly. A well-known example is that of the gene for lactose tolerance; the share of the European population with a "tolerant allele" increased from $5 \%$ to $70 \%$ in less than 5,000 years, due to changes in food habits. In contrast, most Asian populations are lactose intolerant because their cultures had not developed dairy farming. ${ }^{4}$ It is this kind of evolution that I consider here: the race between existing competing alleles. The message is then that in some sense, markets "slow" evolution, by making alleles less loaded with selective pressure. The example of lactose tolerance suggests that trade can have a significant impact on our gene pool if it has been present for say 10,000 years.

This paper's contribution is theoretical, but it may have interesting implications concerning the "nature vs. nurture" debate. For example, there is a controversy on the role played by genes in determining economic success. To simplify, social scientists, looking at income, tend to favor the environment ${ }^{5}$ more than psychometrists, who look at test scores and insist on the genetic determinants of intelligence. ${ }^{6}$. My model suggests that specialization allows to disconnect economic success from general measures of ability, and that the former is less influenced by genes than the latter. One should therefore expect measures of economic success to be less heritable than test scores.

The paper is organized as follows. The next section describes a simplified model of genetic evolution with sexual reproduction. Section 3 introduces natural selection by assuming that genotypes differ by their survival rates; some genotypes are better than others at activities that affect survival. Section 4 shows that under autarky, only the strongest alleles survive in the long-run. Section 5 shows that under trade, a continuum of long-run equi-

\footnotetext{
${ }^{4}$ See e.g. Aoki (1991).

${ }^{5}$ See Becker and Tomes (1986), Ashenfelter and Krueger (1994).

${ }^{6}$ This debate is somewhat captured by the heated "Bell curve" debate in the 1990s. (Herrnstein and Murray, 1995; Devlin et al. 1997; Cawley et al. 1996; Ashenfelter and Rouse (1998)). Note that recent evidence on brain structure, as well as studies of heritability of test scores, suggest that intelligence has substantial genetic determinants. See Bouchard and McGue (1981), and recent studies by Tang et al. (1999), Tsien (2000)). and, Thompson et al. (2001).
} 
libria exist, such that one weak allele has disappeared, but others may be present in strictly positive proportions. It is also shown that the market price of each fitness-enhancing activity is pinned down in a long-run equilibrium, and inversely proportional to the strongest genotype's productivity at that activity. Population size is shown to be larger under trade than autarky, while population growth is the same. Section 6 shows that the model can be extended to study forms of organization that are intermediate between autarky and trade, i.e. 'hunter-gatherer' groups. It is shown that in such a case, as in autarky, only the strongest alleles eventually survive. However, the weaker alleles are eliminated at a slower rate than under autarky. This rate is lower, the larger the size of the group and the more it can replicate the optimal specialization that the market achieves. Section 7 analyzes the transitional dynamics to the long-run equilibrium when the economy is not initially in a long-run equilibrium. It is shown that trade then allows the strongest genotype to achieve even greater fitness; hence along the transition path, trade exacerbates inequality. As a result, the frequency of the strongest alleles replicate at an even higher rate than under autarky. But this process is self-defeating because it has a feedback effect on prices: as the best genotype is more frequent, the price of the goods in which it specializes falls, which eventually makes it possible for the weaker genotypes to survive. Also, convergence to a long-run equilibrium is speeded up when one allows for migration between trading and non trading populations, and migration widens the gap in population size between a trading and a non-trading population. Section 8 introduces environmental shocks that may reverse the fitness advantage of a given allele. A trading population then grows faster than a non-trading one, as specialization helps its members to achieve the highest fitness level in all environments. Section 9 discusses the conditions under which the results can be extended to a more general model of sexual reproduction, referring to a companion paper. Section 10 briefly discusses some qualifications to the results that apply when other phenomena are introduced into the model. 


\section{The model: population genetics}

This section describes the basic features of the model, and its demographic implications. It is a simplified representation of genetic evolution in a heterogeneous population. This haploid population has two chromosomes with a single locus each. These two (unliked) loci are indexed by $i=1,2 .{ }^{7}$ At each locus there can be one of two competing alleles, indexed by $\kappa=H, L$. Consequently, there are 4 possible genotypes, denoted by $H H, H L, L H$ and $L L$. Genotype $\kappa \kappa^{\prime}$ has allele $\kappa$ at locus 1 and allele $\kappa^{\prime}$ at locus 2 .

Each of these genotypes $g$ has a specific survival rate $\varphi_{g}$, which is interpreted as the probability of surviving until mating ${ }^{89}$.

Reproduction is sexual; at any date $t$, people mate randomly and produce $\nu$ offsprings. They inherit their father's (or mother's) allele at locus $i$ with probability 0.5 , independently of which allele is transmitted at the other locus. Random mating makes it easy to compute the genotypic distribution of population at $t+1$ as a function of the distribution at $t$. Denoting by $h_{i}(t)$ the frequency of the $H$ allele at locus $i$ at date $t$, the genotypic distribution of generation $t$ 's offsprings, denoted by $\pi_{g}(t)$, is given by

$$
\begin{aligned}
\pi_{H H}(t) & =h_{1}(t) h_{2}(t), \\
\pi_{H L}(t) & =h_{1}(t)\left(1-h_{2}(t)\right), \\
\pi_{L H}(t) & =\left(1-h_{1}(t)\right) h_{2}(t), \text { and } \\
\pi_{L L}(t) & =\left(1-h_{1}(t)\right)\left(1-h_{2}(t)\right) .
\end{aligned}
$$

Denoting by $N(t)$ the total surviving population at $t$, and by $n_{g}(t)$ its fraction with genotype $g$, we have

$$
n_{g}(t+1) N(t+1)=\nu \varphi_{g} \pi_{g}(t) N(t)
$$

\footnotetext{
${ }^{7}$ This is clearly not realistic but a simplified representation of sexual reproduction. The extension in Saint-Paul (2006) includes the real-world process, where chromosomes come by pairs, as a special case.

${ }^{8}$ or, equivalently, the probability of mating.

${ }^{9}$ Under trade, $\varphi_{g}$ depends on the total distribution of genes, through its effect on prices.
} 
Total population at $t+1$ can then be computed as

$$
N(t+1)=\nu N(t) \sum_{g} \varphi_{g} \pi_{g}(t)
$$

The distribution of alleles is then recovered as

$$
\begin{aligned}
& h_{1}(t+1)=n_{H H}(t+1)+n_{H L}(t+1) \\
& h_{2}(t+1)=n_{H H}(t+1)+n_{L H}(t+1) .
\end{aligned}
$$

As we shall see below, we assume a positive correlation between the presence of an $H$-allele and fitness. Thus we assume:

ASSUMPTION A1:

$$
\begin{aligned}
\varphi_{H H} & \geq \max \left(\varphi_{H L}, \varphi_{L H}\right), \\
\varphi_{L L} & \leq \min \left(\varphi_{H L}, \varphi_{L H}\right) .
\end{aligned}
$$

Most of the results we will derive will be concerned with a stationary equilibrium, i.e. such that $n_{g}(t+1)=n_{g}(t)$, for all $t$ - that is, the distribution of genotypes is invariant, implying that $h_{i}$ is also constant.

This section's central result is then the following lemma:

LEMMA 1 - Assume (A1) holds. Then, in any stationary equilibrium such that $h_{i}>0, \forall i$, one must have

(i) $n_{H L}=n_{L L}=n_{L H}=0$ if $\varphi_{H H}>\varphi_{H L}$ and $\varphi_{H H}>\varphi_{L H}$.

(ii) $n_{L L}=n_{H L} n_{L H}=0$ if $\varphi_{L L}<\varphi_{H H}$

(iii) all surviving genotypes must have the same survival rate, which is the maximum across all genotypes.

Proof - Consider the evolution of $h_{1}$ : $h_{1}(t+1)=\frac{h_{1}(t)\left[\varphi_{H H} h_{2}(t)+\varphi_{H L}\left(1-h_{2}(t)\right)\right]}{h_{1}(t)\left[\varphi_{H H} h_{2}(t)+\varphi_{H L}\left(1-h_{2}(t)\right)\right]+\left(1-h_{1}(t)\right)\left[\varphi_{L H} h_{2}(t)+\varphi_{L L}\left(1-h_{2}(t)\right)\right]}$. 
Note that $\varphi_{L H} \leq \varphi_{H H}$ and that $\varphi_{L L} \leq \varphi_{H L}$. Clearly, one can have $h_{1}(t+1)=h_{1}(t)$ only if (i) $h_{2}(t)=1$ and $\varphi_{L H}=\varphi_{H H}$, or (ii) $0<h_{2}(t)<1$, $\varphi_{H H}=\varphi_{L H}, \varphi_{H L}=\varphi_{L L}$, or (iii) $h_{1}(t)=1$. Similarly, to have $h_{2}(t+1)=h_{2}(t)$ we need (i) $h_{1}(t)=1$ and $\varphi_{H L}=\varphi_{H H}$, or (ii) $0<h_{1}(t)<1, \varphi_{H H}=$ $\varphi_{H L}, \varphi_{L H}=\varphi_{L L}$, or (iii) $h_{2}(t)=1$. The statements in the lemma derive straightforwardly from these observations. Q.E.D.

This lemma tells us that, as long as the $H$-alleles are present in the population, only the best genotypes survive. Furthermore, as (ii) implies, $H L$ and $L H$ cannot simultaneously survive even though they may be as fit as $H H$ - if $L L$ has a strictly lower survival rate than $H H$. This is because they occasionally mate together, thus yielding some $L L$ types which have a lower survival probability. This process tends to drive $L$ alleles out of the gene pool until they have disappeared at at least one locus, thus preventing any $L L$-individuals from arising.

\section{$3 \quad$ Fitness and survival}

We now describe how survival rates are determined. People have a total time endowment equal to 1 . They allocate time between two activities, referred by "f" (fight) and " $d$ " (defence). Furthermore, they have different productivities in each activity, and these productivities are genetically determined. Productivity at the $f$-activity is determined by the gene at locus 1 , and productivity in $d$ is determined by locus 2. More specifically, if the individual has allele $H$ (resp. $L$ ) at locus 1 , his productivity at $f$ is $f_{H}$ (resp. $f_{L}$ ). Similarly, productivity at activity $d$ is $d_{H}\left(\right.$ resp. $d_{L}$ ) for people with allele $H$ (resp. L) at locus 2 .

Consequently, an individual with genotype $g=\kappa \kappa^{\prime}$ chooses his fight and defence levels $f$ and $d$ subject to the following time allocation constraint:

$$
\frac{f}{f_{\kappa}}+\frac{d}{d_{\kappa^{\prime}}}=1
$$


Survival $\varphi$ is then an increasing function of "fitness" $u$, which is increasing in the total amount of $f$ and $d$ activities: $:^{10}$

$$
\varphi_{\kappa \kappa^{\prime}}=\varphi(u(f, d)) ; u_{1}^{\prime}>0, u_{2}^{\prime}>0
$$

We shall assume that having an $H$-allele increases productivity, that is:

$$
f_{H}>f_{L} ; d_{H}>d_{L}
$$

Finally, we assume that people set $f$ and $d$ in order to maximize their fitness. ${ }^{11}$ Thus they maximize $u(f, d)$ subject to the time allocation constraint (1). In order to avoid analytical problems we shall assume that $u$ is concave, and satisfies the following conditions:

$$
\begin{aligned}
& \lim _{f \rightarrow 0} \frac{u_{1}^{\prime}\left(f, d_{0}\right)}{u_{2}^{\prime}\left(f, d_{0}\right)}=+\infty, \quad d_{0}>0 \\
& \lim _{d \rightarrow 0} \frac{u_{2}^{\prime}\left(f_{0}, d\right)}{u_{1}^{\prime}\left(f_{0}, d\right)}=+\infty, \quad f_{0}>0 .
\end{aligned}
$$

This assumption, which is standard in economics, means that the first unit of each activity is infinitely useful (relative to the other activity), so that if, say, $f=0$ and $d>0$, one is willing to reduce one's consumption of $d$ and to increase one's consumption of $f$, regardless of prices. In other words, the two activities must be sufficiently "complementary".

\footnotetext{
${ }^{10}$ The distinction between fitness and survival is immaterial; maximizing $u$ is like maximizing $\varphi$. The extra notation is used just to avoid manipulating functions that can only take values between 0 and 1 , as $\varphi$ should.

${ }^{11}$ By bluntly making this assumption, we depart from biology and enter economics. A biologist would ask why people should behave like that, and would probably assume the existence of a gene for such behavior, and try to show that it drives out genes for alternative behaviors. This "as if" argument is out of the scope of this paper, and we directly assume maximization of fitness. See Hirschleifer (1977) and Robson (2001) for discussions.
} 
To get analytical solutions we shall often use a Leontief fitness function ${ }^{12}$ :

$$
u(f, d) \equiv \min (f, d)
$$

\section{Autarky}

The preceding section describes an economy without trade. To get the longrun composition of the population is quite simple: The $H H$ type has a more favorable time allocation contraint. Therefore, it is able to achieve a strictly greater fitness. In the Leontief case, for example, the fitness of genotype $\kappa \kappa^{\prime}$ is

$$
u_{\kappa \kappa^{\prime}}^{A}=\frac{f_{\kappa} d_{\kappa^{\prime}}}{f_{\kappa}+d_{\kappa^{\prime}}},
$$

where superscript $A$ stands for Autarky. The corresponding survival rate is $\varphi_{\kappa \kappa^{\prime}}^{A}=\varphi\left(u_{\kappa \kappa^{\prime}}^{A}\right)$. Then, by virtue of Lemma $1, H H$ must be the only remaining type in the long run.

PROPOSITION 1 - The solution to people's maximization problem satisfies (A1) with strict inequalities. Consequently, in any stationary equilibrium with a positive supply of $H$ alleles at each locus:

$$
n_{H H}=1 ; n_{H L}=n_{L H}=n_{L L}=0
$$

Proof - Straightforward by application of Lemma 1.Q.E.D.

In the long run, the strongest gene is "fixed" at both loci. The weaker genes have disappeared. That is in conformity with basic principles of natural selection.

\section{Trade}

I now introduce the possibility of trade among people and derive its implications for the long-run composition of the population. I now assume that

\footnotetext{
${ }^{12}$ It does satisfy (A2) in an extended sense, since the denominators are zero in both cases.
} 
$f$ and $d$, instead of being activities, are tradeable goods. At any date their price is denoted by $p_{f}$ and $p_{d}$, and it is convenient to normalize this price vector so that

$$
p_{f}+p_{d}=1
$$

An equilibrium is then determined the standard way. Each individual of genotype $g=\kappa \kappa^{\prime}$ determines his allocation of time by maximizing income:

$$
\max p_{f} f^{S}+\left(1-p_{f}\right) d^{S}
$$

Subject to the time allocation constraint:

$$
\frac{f^{S}}{f_{\kappa}}+\frac{d^{S}}{d_{\kappa^{\prime}}}=1
$$

This determines his supply to the market of goods $f$ and $d, f^{S}\left(g, p_{f}\right)$ and $d^{S}\left(g, p_{f}\right)$, as well as his total income $R\left(g, p_{f}\right)=p_{f} f^{S}+\left(1-p_{f}\right) d^{S}$.

People purchase quantities $f^{D}$ and $d^{D}$ of each good on the market, by maximizing $u\left(f^{D}, d^{D}\right)$ subject to

$$
p_{f} f^{D}+\left(1-p_{f}\right) d^{D}=R\left(g, p_{f}\right)
$$

This determines the individual demand functions $f^{D}\left(g, p_{f}\right)$, and $d^{D}\left(g, p_{f}\right)$, and the resulting fitness $u\left(f^{D}\left(g, p_{f}\right), d^{D}\left(g, p_{f}\right)\right)$. An economic equilibrium at $t$ is an allocation and a price vector which are solution to these optimization problems and such that markets clear, i.e. ${ }^{13}$

$$
\sum_{g} n_{g}(t) f^{D}\left(g, p_{f}\right)=\sum_{g} n_{g}(t) f^{S}\left(g, p_{f}\right)
$$

We are now interested in how economic forces affect the long-run genetic composition of the population. For this we introduce the concept of a

\footnotetext{
${ }^{13}$ By Walras' law, if this holds, then the market for $d$ is also in equilibrium. Standard results (Debreu, 1959) tell us that, given the current distribution of genotypes $\left\{n_{g}\right\}$, an equilibrium exists and is Pareto optimal, in that an agent's fitness can't be increased without reducing another agent's fitness.
} 
long-run equilibrium, which is a situation where the economy is in economic equilibrium and the distribution of genotypes in the population is stationary. The two are interrelated because the economic equilibrium determines the level of fitness of each genotype, which in turn affects its population genetics.

DEFINITION - A Long-Run Equilibrium (LRE) is an allocation $\left(f_{g}^{S}, d_{g}^{S}, f_{g}^{D}, d_{g}^{D}\right)$, a price vector $p_{f}$, and a genotypic distribution $\left\{n_{g}\right\}$ such that

(i) Markets clear, i.e. $f_{g}^{S}=f^{S}\left(g, p_{f}\right), f_{g}^{D}=f^{D}\left(g, p_{f}\right), d_{g}^{S}=d^{S}\left(g, p_{f}\right), d_{g}^{D}=$ $d^{D}\left(g, p_{f}\right)$, and (4) holds.

(ii) The genotypic distribution is stationary, i.e. $n_{g}(t+1)=n_{g}(t), \forall g$.

We now turn to the central result of this section, which characterizes the properties of an LRE. We first state it formally and prove it, and then discuss it.

PROPOSITION 2 - In any LRE

(i) The price of $f$ must be equal to

$$
p_{f}=\frac{d_{H}}{d_{H}+f_{H}}
$$

(ii) The L-allele has disappeared at at least one locus.

Proof of (i) - This price makes the $H H$ type indifferent between supplying $f$ and supplying $d$. Suppose, say, $p_{f}>\frac{d_{H}}{d_{H}+f_{H}}$. Then $H H$ only supplies $f$, and so does $H L$. As for $L H$, his maximum income does not exceed $\max \left(p_{f} f_{L},\left(1-p_{f}\right) d_{H}\right)<\max \left(p_{f} f_{L}, \frac{d_{H} f_{H}}{d_{H}+f_{H}}\right)<p_{f} f_{H}$. Consequently, $\varphi_{L H}<\varphi_{H H}$. Next, Lemma 1, (iii), implies that both $L L$ and $L H$ must have disappeared in equilibrium. But, then total supply of good $d$ is zero, which can't be true because of conditions (A2). A similar line of reasoning holds if $p_{f}<\frac{d_{H}}{d_{H}+f_{H}}$.

Proof of (ii) - The $L L$ type has a strictly lower income than $H H$, therefore $\varphi_{L L}<\varphi_{H H}$. Applying (ii) in lemma 2 does the rest. Q.E.D. 
Part (i) of Proposition 2 is striking. It tells us that in an LRE, the price vector is entirely pinned down by the productivity levels of the $H$ alleles, regardless of the shape of the fitness function $u$ and of the composition of the population. This is a property of any LRE but not of a situation which would just be an economic equilibrium. In other words, $p_{f}$ may transitorily differ from $\frac{d_{H}}{d_{H}+f_{H}}$ but in the long-run it has to be equal to it.

The intuition is as follows: if the $H H$ type were not indifferent between the two goods, it would specialize in one of them, and any type which supplies the other would be strictly worse-off, i.e. less fit, than $H H$, since its productivity at doing it is at most as high as that of $H H$, which strictly prefers not supplying it. Then, all suppliers of this good gradually disappear relative to the rest of the population, and this cannot be in an LRE.

As a corollary, the $H H$ type has no gains from trade in an LRE, because the relative price $p_{f} / p_{d}$ is equal to its marginal rate of transformation between the two activities. For this to be the case it must be, as shown below, that the $H$ allele is sufficiently abundant.

Part (ii) tells us that one of the two $L$-alleles has to disappear, because of the $L L$ type acting as a genetic well. What is important, however, is that only one weak gene has to disappear, whereas all of them were eventually eliminated under autarky. Trade allows weaker people to specialize in the activity where they can match the best, thus making their genetic deficiencies irrelevant for survival. Consequently, these 'inferior' genes are passed to the next generation with the same frequency as 'superior' ones, and are no longer eliminated in the long run. The $L L$-type, on the other hand, has an absolute disadvantage in all activities and as long as mating between people with an $L$-gene produces some $L L$ 's, the $L$ genes gradually disappear in relative terms. But this process stops when $H$ is fixed at one of the two loci, since no new $L L$ is then produced.

It is easy to construct equilibria where the $L$-allele survives at one locus. To do this let us simply take the Leontief fitness function $u(f, d) \equiv \min (f, d)$. Let us construct an LRE where $n_{H H}>0$ and $n_{H L}>0$. Since $p_{f}+p_{d}=1$, the 
maximum fitness of an agent with income $R$, at $d=f$, is equal to $R$. Thus fitness under trade for genotype $g$ and price $p_{f}$ is simply $u_{g}^{T}\left(p_{f}\right)=R\left(g, p_{f}\right)$.

Since, at $p_{f}=\frac{d_{H}}{d_{H}+f_{H}}$, genotype $H H$ is indifferent between the two activities, $H L$ must specialize in $f$. Thus its supply of $f$ is $n_{H L} f_{H}$, and its income is $R\left(H L, \frac{d_{H}}{d_{H}+f_{H}}\right)=\frac{d_{H} f_{H}}{d_{H}+f_{H}}$, which also equals its demand for both goods:

$$
f^{D}(H L)=d^{D}(H L)=\frac{d_{H} f_{H}}{d_{H}+f_{H}}
$$

The income of $H H$ is also equal to $\frac{d_{H} f_{H}}{d_{H}+f_{H}}$ and its demand is therefore the same:

$$
f^{D}(H H)=d^{D}(H H)=\frac{d_{H} f_{H}}{d_{H}+f_{H}}
$$

To get an equilibrium it must be that the total demand for $f$ exceeds what $H L$ is supplying. The difference is then supplied by $H H$. Thus it must be that:

$$
\frac{d_{H} f_{H}}{d_{H}+f_{H}}\left(n_{H H}+n_{H L}\right) \geq n_{H L} f_{H}
$$

or equivalently ${ }^{14}$

$$
n_{H L} \leq \frac{d_{H}}{d_{H}+f_{H}}
$$

This condition is necessary and sufficient for an economic equilibrium. Then noting that $H L$ and $H H$ have the same mortality rate, one can trivially check that the stationarity conditions $n_{g}(t+1)=n_{g}(t)$ are satisfied. In fact any initial distribution satisfying $n_{L H}=n_{L L}=0$ and (6) will indefinitely reproduce itself, without any transitional dynamics.

Thus, as long as the proportion of $H L$ in the population is not too high, the economy can be in an LRE with stationary proportions of each type.

\footnotetext{
${ }^{14}$ Similarly an LRE with $H H$ and $L H$ in the populatio can be constructed, a necessary and sufficient condition is

$$
n_{L H}<\frac{f_{H}}{d_{H}+f_{H}}
$$
}


In such an equilibrium, fitness is simply equal to .

$$
u_{H L}^{T}=u_{H H}^{T}=\frac{d_{H} f_{H}}{d_{H}+f_{H}}=u_{H H}^{A} .
$$

Note also that the RHS of (6) is greater, the greater the maximum productivity level in the $d$-activity relative to the $f$-activity. When $d_{H} / f_{H}$ is large, only a few people are needed to produce society's demand for the $d$-good. Since all of these people must be of genotype $H H$ in equilibrium, equilibrium exists if $n_{H H}$ is large enough relative to the required number of people who must produce $d$. This is more likely to be the case, the smaller this number, i.e. the greater $d_{H} / f_{H}$.

If $n_{H H}$ is initially to small, the $H H$ types will have positive gains from trade and will achieve higher fitness than the $H L$ type. The frequency of the $H$ allele in the population will rise until (6) is satisfied. These transitional dynamics are studied in Section 7 below.

While Proposition 2 has characterized the equilibrium in terms of prices and genotypic composition, the following one compares trade and autarky in terms of total population. ${ }^{15}$

PROPOSITION 3 - (i) The long-run population growth rate is the same under trade and autarky

(ii) For given initial conditions, population at any subsequent date is larger under trade than autarky.

Proof - (i) follows from the observation that $u_{H L}^{T}=u_{H H}^{T}=u_{H H}^{A}$. Trade and autarky yield the same population growth rate equal to $\nu \varphi_{H H}^{A}$.

To prove (ii), just note that for any given type, fitness is always higher under trade than under autarky - even if the economy is not currently in an LRE - since each genotype could replicate its autarkic allocation of time. Q.E.D.

\footnotetext{
${ }^{15}$ Proposition 3 clearly ignores phenomena such as the demographic transition, when increased fitness may mean lower population growth (See Galor and Moav (2000) for an analysis). Such effects could be reintroduced by endogenizing birth rates.
} 


\section{6 'Hunter-gatherers'}

One may criticize the preceding analysis on the grounds that the autarky metaphor is too stylized, in that one does not observe Robinson Crusoe human societies in reality. Rather, one observes small tribes with egalitarian resource sharing (hereafter nick-named "hunter-gatherers", HG) ${ }^{16}$, a more advanced form of organization than mere autarky.

In this section, we extend the analysis to such groups. We do so in such a way that autarky and trade are special cases. We show that except for large HG groups with efficient specialization (which is equivalent to trade), the $L$ alleles are eventually eliminated, as in the pure autarky case. That may be due either to inefficient specialization or to small group size. Let us discuss these effects in turn.

First of all, because of the egalitarian allocation of resources, the HG society lacks a mechanism for inducing its members to specialize efficientlycontrary to the market which rewards people for choosing the most productive activity. People will tend to select the least unpleasant activity rather than the most productive one, and therefore will not specialize according to comparative advantage ${ }^{17}$. That effect will make it impossible for the members of the tribe to achieve the maximum fitness level that optimal specialization would yield. As a result, tribes with a higher frequency of high alleles will achieve a higher fitness, as in the autarky case; in the long-run, high alleles will prevail. ${ }^{18}$

\footnotetext{
${ }^{16}$ See, for example Boehm $(1993,2001)$ for an analysis of egalitarianism in HG tribes.

${ }^{17}$ If any consensus emerges from the literature, it is that some specialization takes place in HG societies, but that this is much more rudimentary than the one which arises in complex trading societies. One mostly observes specialization according to sex and/or age. See Jochim (1988), Watanabe (1983), Lee and Devore (1968), Dahlberg (1981) and Jones (1996). Therefore, task assignment is not random but far from efficient either. That makes sense: in the absence of a market mechanism, the group leaders can improve efficiency only by assigning tasks on the basis of some obvious characteristic, such as sex. All this suggests that we expect real-world HG societies to be closer to "autarky" than to the "trading" population.

${ }^{18}$ Note that it is not a group selection argument: individual fitness is an increasing function of the average frequency of high alleles in the individual's tribe.Because of egalitarian sharing, individuals with an $H$-allele do not achieve a higher fitness level than individuals
} 
Building on the above results, let us concentrate on the case where allele $H$ is fixed at locus 1 (the $f$ activity). Assume total population is large, equal to $N(t)$ at date $t$. People are split in tribes of constant size $Q<<N$. Each tribe is characterized by the number $Q_{H}$ of individuals who have an $H$-allele at locus $d$. Consider the extreme case where the tribe is unable to allocate people to tasks on the basis of their productivity. Say it asks from each individual a uniform contribution $f$ to the $f$-activity, while his contribution to the $d$-activity is determined residually ${ }^{19}$. That is, an, individual $j$ with a $\kappa$-allele at locus 2 will provide $d(j)=\left(1-f / f_{H}\right) d_{\kappa}$ of the $d$-activity. The aggregate amounts of $f$ and $d$ are then shared equally between the members of the tribe. Let us assume again a Leontief fitness function $\min (f, d)$ and that the tribe manages to calibrate $f$ so as to optimally yield an amount $d=f$ for each member. As $d=\frac{Q_{H}}{Q}\left(1-\frac{f}{f_{H}}\right) d_{H}+\frac{Q-Q_{H}}{Q}\left(1-\frac{f}{f_{H}}\right) d_{L}$, this yields a fitness equal to:

$$
d=f=f_{H} \frac{\frac{Q_{H}}{Q} d_{H}+\left(1-\frac{Q_{H}}{Q}\right) d_{L}}{f_{H}+d_{H} \frac{Q_{H}}{Q}+\left(1-\frac{Q_{H}}{Q}\right) d_{L}}=u_{0}\left(Q_{H} / Q\right)
$$

That is clearly strictly increasing with $Q_{H}$.

Consider now the more interesting case where the tribe manages to allocate people efficiently to the two tasks. It can then replicate the outcome of a market economy and reach the same fitness level as if all individuals had an $\mathrm{H}$-allele. However, that is only true for tribes such that (6) holds. Tribes for which (6) does not hold will have a lower fitness, and these will be the tribes with a higher frequency of an $L$-allele.

Let us see this more precisely. An optimal allocation of effort within a tribe must be such that genotype $\kappa \kappa^{\prime}$ specializes in $f$ if $d_{\kappa^{\prime}} / f_{\kappa}<\delta$, in $d$ if $d_{\kappa^{\prime}} / f_{\kappa}>\delta$, and can produce both commodities if $d_{\kappa^{\prime}} / f_{\kappa}=\delta$, where $\delta$ is the endogenous comparative advantage threshold. Since, at the optimum, $d=f$,

with an L-allele in the same tribe. However, $H$-alleles eventually drive-out $L$-alleles in the long-run because of a composition effect: they are overrepresented in the tribes that achieve a higher fitness.

${ }^{19}$ Any other assumption which deviates from efficient specialization would yield the same results. 
generically, some individuals must produce both commodities. Thus there are only two possibilities: either $\delta=d_{H} / f_{H}$ or $\delta=d_{L} / f_{H}$. The first case prevails if $Q_{H} f_{H}>\left(Q-Q_{H}\right) d_{H}$, i.e.

$$
\frac{Q_{H}}{Q}>\frac{f_{H}}{f_{H}+d_{H}}=\bar{q}
$$

and the resulting fitness for each member of the tribe is then ${ }^{20}$

$$
f=d=\frac{f_{H} d_{H}}{f_{H}+d_{H}}=u_{1}\left(Q_{H} / Q\right)
$$

Thus, under condition (8), which is equivalent to (6), the tribe replicates the market equilibrium, which yields the highest fitness to all members, independently of the proportion of $H$-alleles in the tribe. Conversely, if (8) is violated, then $\delta=d_{L} / f_{H}$, and the tribe achieves the following fitness level: ${ }^{21}$

$$
f=d=\frac{\frac{Q_{H}}{Q} f_{H} d_{H}+\left(1-\frac{Q_{H}}{Q}\right) f_{H} d_{L}}{f_{H}+d_{L}}=u_{1}\left(Q_{H} / Q\right)
$$

In this zone, fitness is again an increasing function of $Q_{H} / Q$.

To summarize:

- If the allocation of tasks is "random", fitness is an increasing function $u_{0}$ of $Q_{H} / Q$;

- If it is efficient, it is an increasing function $u_{1}$ of $Q_{H} / Q$ up to a threshold, $\bar{q}$, then stays constant at its maximum level.

Let us now check that in both cases, the $L$-allele eventually disappears from the population. Let us assume that mating is random and exogamic (i.e. one does not mate in one's tribe). Tribes are formed randomly at each

\footnotetext{
${ }^{20}$ Denoting by $\kappa(j)$ the allele at locus 2 of individual $j$, the common individual fitness level derives from the unique allocation of effort such that $f=d$, that is $Q . d=$ $\sum_{\kappa(j)=H} d(j)=Q . f=\sum_{\kappa(j)=H} f(j)+f_{H}\left(Q-Q_{H}\right)$

$=f_{H} Q_{H}-Q . d \cdot \frac{f_{H}}{q_{H}}+d_{H}\left(Q-Q_{H}\right)$, which yields $(9)$.

${ }^{21}$ To equate $f$ and $d$, one must have in this case $f \cdot Q=\sum_{\kappa(j)=L} f(j)=d \cdot Q=Q_{H} d_{H}+$ $\sum_{\kappa(j)=L} d(j)$

$=Q_{H} d_{H}+d_{L}\left(Q-Q_{H}\right)-\frac{d_{L}}{f_{H}} f \cdot Q$, which yields (10).
} 
new generation. Therefore, the frequency of $Q_{H}$ in the population is given by the binomial distribution:

$$
\rho\left(Q_{H} ; h_{2}(t)\right)=\frac{C_{Q}^{Q_{H}}}{2^{Q}} h_{2}(t)^{Q_{H}}\left(1-h_{2}(t)\right)^{Q-Q_{H}},
$$

where $h_{2}(t)$ is again the frequency of the $H$-allele at locus 2. The survival rate of an individual in a tribe with $Q_{H}$ is $\varphi\left(u_{k}\left(Q_{H} / Q\right)\right)$ which, in the two cases we have discussed $(k=0,1)$, is nondecreasing and strictly increasing for $Q_{H} / Q<\bar{q}$. Consequently, the frequency of the $h$-allele in the distribution of offsprings is

$$
h_{2}(t+1)=\frac{\sum_{Q_{H}=0}^{Q} \rho\left(Q_{H} ; h_{t}\right) \frac{Q_{H}}{Q} \varphi\left(u_{k}\left(Q_{H} / Q\right)\right)}{\sum_{Q_{H}=0}^{Q} \rho\left(Q_{H} ; h_{t}\right) \varphi\left(u_{k}\left(Q_{H} / Q\right)\right)}=\psi\left(h_{2}(t)\right)
$$

This quantity is clearly larger than $h_{2}(t)=\sum_{Q_{H}=0}^{Q} \rho\left(Q_{H} ; h_{2}(t)\right) \frac{Q_{H}}{Q}$. Thus $h_{2}(t)$ grows over time. Furthermore, since $\rho\left(Q_{H} ; h_{2}(t)\right)>0$ for all $Q_{H}$, and $\varphi\left(u_{k}\left(Q_{H} / Q\right)\right)$ is strictly increasing over some range, one has $\psi(h)>h$ for all $h \in(0,1)$. Thus $h=0$ and $h=1$ are $\psi$ 's only fixed points. Therefore, $h_{2}(t)$ monotonically converges to 1 .

L-alleles are eventually eliminated from HG societies even though these implement efficient specialization within a tribe. The reason is that the law of large numbers does not apply to the distribution of $Q_{H}$. At each date, a positive fraction of individuals end up in tribes such that $Q_{H} / Q<\bar{q}$. These individuals achieve lower fitness and are also more likely to have an $L$-allele. Consequently, this allele eventually disappears. ${ }^{22}$

In some sense, this section has studied the effects of living in large groups. The greater $Q$, the more the distribution of $\rho\left(Q_{H} ; h_{2}(t)\right)$ resembles a mass point at $Q_{H}=h_{2}(t)$. If the economy is in a zone where $h_{2}(t)>\frac{f_{H}}{f_{H}+d_{H}}$, the $L$-allele will therefore be eliminated at a lower rate, the larger the size of the group $Q$. The "trade" case analyzed above is a special case when $Q$ becomes large, while autarky is the special case associated with $Q=1$. Note

\footnotetext{
${ }^{22}$ Intuitively, the result would still hold under endogamy: because of genetic drift, tribes such that $h_{2}(t)<1$ would regularly end up below the threshold $\bar{q}$, and they will eventually be replaced by tribes such that $h_{2}=1$.
} 
however that this reasoning ignores the coordination problems involved in allocating people to the right tasks as $Q$ becomes large; markets are indeed an institution which solves these problems.

To conclude this section: the $L$ allele will eventually be eliminated from small HG group, regardless of how efficiently they specialize.

\section{Transitional dynamics}

Section 5 has studied the long-run equilibrium of a trading population and shown that it is compatible with survival of the L-allele at one locus. In this section, I analyse the transitional dynamics to an LRE. I will again assume that $H$ is fixed at locus 1 , but that (6) is initially violated.

\subsection{The economic equilibrium in a transition}

When (6) is violated, the only possibility for an equilibrium is that $H H$ provides $d$ and $H L$ is indifferent between providing $f$ and $d$. Consequently the price level must be

$$
p_{f}=\frac{d_{L}}{f_{H}+d_{L}}
$$

The $H L$ type achieves a fitness level $p_{f} f_{H}=p_{d} d_{L}=\frac{f_{H} d_{L}}{f_{H}+d_{L}}$. It has no gains from trade and achieves the same fitness as in autarky. The $H H$ type has a fitness level $p_{d} d_{H}=\frac{f_{H} d_{H}}{f_{H}+d_{L}}>\frac{d_{H} f_{H}}{d_{H}+f_{H}}$. It has positive gains from trade and achieves a greater fitness level than under autarky.

The dynamics are simple: the frequency of the $H$ allele will increase; hence $n_{H L}$ will fall, until the first date when (6) is satisfied. $n_{H L}$ will then typically be a bit above the threshold, and would stay there; but that is due to the discrete time structure of the model, and it is easy to see that (6) would be matched with equality under continuous time. When the economy enters the LRE, fitness drops from $\frac{d_{H} f_{H}}{d_{L}+f_{H}}$ to $\frac{d_{H} f_{H}}{d_{H}+f_{H}}$ for the $H H$ type, and jumps from $\frac{d_{L} f_{H}}{d_{L}+f_{H}}$ to $\frac{d_{H} f_{H}}{d_{H}+f_{H}}$ for the $H L$ type. 
Comparing trade and autarky during the transition, the $H$-allele replicates faster under trade, but that eventually eliminates its economic advantage and the economy settles in an LRE with $n_{H L}>0$, while selection continues to operate under autarky. These transitional dynamics are illustrated on Figure 1: there is a first phase of rapid increase in the frequency of the $\mathrm{H}$ allele, and a second phase where the economy is an LRE where both types have the same fitness.

\subsection{Inequality}

By construction, the above model rules out any inequality in income and fitness between individuals in a long-run equilibrium, regardless of the institutional settings. That is because inequality can only come from differences in genotypes: if there were inequality, more successful genes would replicate at a higher rate and one would not be in a long-run equilibrium.

Yet, more advanced societies typically generate more inequality than primitive ones. In some sense, that should not come as a surprise: the potential for generating inequality is much smaller if one is close to the subsistence level. But can we reconcile that observation with the mechanism discussed in this paper?

The preceding subsection implies that inequality may arise as a transitional phenomenon. Along the transition path just described, $H H$ has a higher income and fitness under trade than under autarky, while $H L$ has the same fitness level. Therefore, inequality is higher under trade than under autarky. As for the egalitarian HG society, inequality is even smaller than under autarky: within-tribe inequality is equal to zero by construction, and per-capita tribe income spans the $\left[\frac{f_{H} d_{L}}{f_{H}+d_{L}}, \frac{f_{H} d_{H}}{f_{H}+d_{H}}\right]$ as $Q_{H}$ varies from 0 to $Q$, whereas under autarky individual income is equal to one of these two bounds.

Second, inequality may persist if it is unrelated to genotype. Suppose that some individuals are lucky and with some small probability $p$ can get hold of a large amount of resources in the $f$ activity; call $F$ that amount. Assume $p$ is the same for all genotypes. Under autarky, the effect of luck on fitness 
is limited by concavity in the $u(.,$.$) function. In the extreme Leontief case,$ fitness can only increase up to $d_{\kappa}$, the individual's endowment in the other activity. In the HG society, the prize $F$ is shared between all the members of the tribe. If tasks are allocated optimally, then each member's fitness typically goes up but cannot exceed $\left(1-\frac{Q_{H}}{Q}\right) d_{L}+\frac{Q_{H}}{Q} d_{H} \cdot{ }^{23}$ Under trade, the individual's fitness is equal to $p_{f} F+\max \left(p_{f} f_{\kappa}+\left(1-p_{f}\right) d_{\kappa}\right)$. It therefore goes up without bounds with $F$. Trade can therefore generate a much larger level of inequality than autarky and HG societies, yet as long as luck is unrelated to genotypes (which must be for inequality to be persistent in an LRE), the model's conclusions are unchanged.

\subsection{Migration}

It is interesting to discuss how the transitional dynamics are changed if one allows for migration between trading and non-trading populations. In the logic of this paper, trade is a cultural trait associated with a population, not a genetic trait associated with individuals. Since trade makes people at least as well-off as autarky (or HG tribes), migration would tend to take place from autarkic populations to those who trade.

A natural way to think of migration is to assume that the fraction of the population which migrates is larger, the larger the fitness gain associated with such a move. In an LRE, fitness is the same in a trading and in a non trading population (with only $H H$ genotypes), so that there should be no net migration between the two populations. ${ }^{24}$ During the transition, however,

\footnotetext{
${ }^{23}$ Straightforward computations along the lines of the previous section allow to prove that. There are three cases:

1. If $F<Q_{H} d_{H}-\left(Q-Q_{H}\right) f_{H}$, then $\delta=d_{H} / f_{H}$ and the common fitness level $u$ is equal to $f=d=u=\frac{d_{H}\left(f_{H}+F / Q\right)}{f_{H}+d_{H}} \leq \frac{d_{H} Q_{H}}{Q}$.

2. If $Q_{H} d_{H}-\left(Q-Q_{H}\right) f_{H} \leq F \leq\left(Q-Q_{H}\right) d_{L}+Q_{H} d_{H}$, then $\delta=d_{L} / f_{H}$ and the common fitness level is equal to $f=d=u=\frac{\left(1-Q_{H} / Q\right) d_{L} f_{H}+\left(Q_{H} / Q\right) d_{H} f_{H}+d_{L} F / Q}{d_{L}+f_{H}} \leq$ $\left(1-\frac{Q_{H}}{Q}\right) d_{L}+\frac{Q_{H}}{Q} d_{H}$.

3. If $F>\left(Q-Q_{H}\right) d_{L}+Q_{H} d_{H}$, then $f=F / Q>d=u=\left(1-\frac{Q_{H}}{Q}\right) d_{L}+\frac{Q_{H}}{Q} d_{H}$.

${ }^{24}$ Furthermore, if a type migrates to a trading population, its fitness does not exceed that of the resident types. Therefore, the LRE passes the invasibility test discussed in Mylius and Diekmann (1995).
} 
interesting effects arise, to the extent that $H L$ types are still present in the non-trading population. Suppose that (6) holds at a point in time. Then the price level is $p_{f}=d_{H} /\left(f_{H}+d_{H}\right)$, and the $H H$ type achieves the same fitness level as under autarky. Thus we expect only $H L$ types to migrate from a non-trading to a trading population. But that increases the relative frequency of $L$ alleles in the trading population, so that (6) may eventually be violated. On the other hand, if it is violated with strict inequality, then the $H H$ type has gains from trade, and not the $H L$ type. There will therefore be an inflow of $H H$ types. Thus, in the medium run, we expect migration to affect the gene pool of the trading population in such a way that (6) holds with equality. The frequency of the $H$ allele in the trading population is therefore entirely pinned down by this condition.

When (6) holds with equality, relative prices are no longer determinate. Provided $\frac{d_{L}}{d_{L}+f_{H}}<p_{f}<\frac{d_{H}}{d_{H}+f_{H}}$, the $H H$ type will entirely specialize in the $d$ activity and the $H L$ type in the $f$ activity. Markets clear regardless of $p_{f}$ because of the equality in (6). How is then $p_{f}$ determined? It must take the only value such that migrations are compatible with that equality. To see this more precisely, suppose that a fraction $m_{g}(t)$ of people with genotype $g$ migrates from the autarkic to the trading population every period. Assume it is an increasing function of the differences in fitness between the two populations

$$
m_{g}(t)=\gamma\left(u_{g}^{T}\left(p_{f}(t)\right)-u_{g}^{A}\right)
$$

where $\gamma()$ is such that $\gamma() \geq 0, \gamma(0)=0, \gamma()<1$, and $\gamma^{\prime}()>0$. While $u_{g}^{A}$ is given by (2), the specialization pattern implies that:

$$
\begin{aligned}
& u_{H H}^{T}\left(p_{f}\right)=\left(1-p_{f}\right) d_{H} ; \\
& u_{H L}^{T}\left(p_{f}\right)=p_{f} f_{H} ;
\end{aligned}
$$

Using Section 2, we get that the non trading population evolves according 
to

$$
\begin{aligned}
N^{A}(t) n_{H L}^{A}(t) & =\nu \varphi_{H L}^{A} N^{A}(t-1) n_{H L}^{A}(t-1)\left(1-m_{H L}(t)\right) ; \\
N^{A}(t) n_{H H}^{A}(t) & =\nu \varphi_{H H}^{A} N^{A}(t-1) n_{H H}^{A}(t-1)\left(1-m_{H H}(t)\right) .
\end{aligned}
$$

The trading population evolves according to

$$
\begin{aligned}
N^{T}(t) n_{H L}^{T}(t)= & \nu \varphi\left(u_{H L}^{T}\left(p_{f}\right)\right) N^{T}(t-1) n_{H L}^{T}(t-1) \\
& +m_{H L}(t) \nu \varphi_{H L}^{A} N^{A}(t-1) n_{H L}^{A}(t-1) \\
N^{T}(t) n_{H H}^{T}(t)= & \nu \varphi\left(u_{H H}^{T}\left(p_{f}\right)\right) N^{T}(t-1) n_{H H}^{T}(t-1) \\
& +m_{H H}(t) \nu \varphi_{H H}^{A} N^{A}(t-1) n_{H H}^{A}(t-1)
\end{aligned}
$$

Migration flows must adjust so as to maintain equality in (6), that is equivalent to

$$
\frac{d_{H}}{f_{H}}=\frac{n_{H L}^{T}(t)}{n_{H H}^{T}(t)}
$$

which because of (13) and (14) defines a unique value for $p_{f}(t)$.

Asymptotically, the ratio $n_{H L}^{A}(t) / n_{H H}^{A}(t)$ has to fall to zero, since the $H L$ type achieves lower fitness in autarky. ${ }^{25}$ For (15) to hold, it must be that $m_{H H}(t)$ asymptotically goes to zero, meaning that $p_{f}(t)$ goes to $d_{H} /\left(f_{H}+d_{H}\right)$. Thus one converges to the above described LRE where (6) holds with equality.

In the short run, the initial conditions may be such that (15) cannot hold. For example, if $\frac{\varphi\left(\frac{f_{H} d_{L}}{f_{H}+d_{L}}\right) n_{H L}^{T}(t-1) N^{T}(t-1)}{\varphi\left(\frac{f_{H} d_{H}}{f_{H}+d_{L}}\right) N^{T}(t-1) n_{H H}^{T}(t-1)+\gamma\left(\frac{f_{H} d_{H}}{f_{H}+d_{L}}-\frac{f_{H} d_{H}}{f_{H}+d_{H}}\right) \varphi_{H H}^{A} N^{A}(t-1) n_{H H}^{A}}>\frac{d_{H}}{f_{H}}$,

then the economic equilibrium is such that $p_{f}=\frac{d_{L}}{f_{H}+d_{L}}$. One has $u_{H L}^{T}\left(p_{f}\right)=$ $u_{H L}^{A}$ and $m_{H L}=0$. Only the $H H$ type migrates to the trading economy,

\footnotetext{
${ }^{25}$ The only alternative is a constantly higher migration rate for the $H H$ type. That is, to have $n_{H L}^{A}(t) / n_{H H}^{A}(t)>K$, we need $m_{H H}(t)>\frac{y-1}{y}+m_{H L}(t)$, with $y=\varphi\left(u_{H H}^{A}\right) / \varphi\left(u_{H L}^{A}\right)>$ 1. But then, that implies that population in autarky shrinks without bounds relative to the trading population. That makes it impossible for migration to sustain a constant $n_{H L}^{T} / n_{H H}^{T}$ ratio with migration, unless $u_{H L}^{T}=u_{H H}^{T}$. But in such a situation $m_{H H}(t)$ becomes equal to zero, which is a contradiction.
} 
which speeds up growth in the frequency of the $H$ allele there, while this may actually (temporarily) favor the $L$ allele in the autarkic population. ${ }^{26}$

Figure 2 plots the transition to an LRE for the trading population, under migration vs. no migration, when the initial density of the $H$ allele is low enough, so that (16) initially holds. Under migration, one converges more quickly to the critical density of $L$ genes, $\frac{d_{H}}{f_{H}+d_{H}}$. As for the non-trading population (Figure 3), migration initially slows or reverts the elimination of the $L$ allele and then accelerates it. There is also a qualitative difference in that, under trade, the $H L$ type achieves the same fitness level as the $H H$ type in finite time absent migration, while convergence is only gradual under migration (Figure 4).

It is also obvious that the relative population size of the non-trading population in the long-run is lower under migration. A less obvious question is: can it fall to zero? Interestingly, one can prove that it is not the case (See Appendix). Migration can never be strong enough to eliminate the nontrading population absent environmental shocks: as $p_{f}$ converges to $\frac{d_{H}}{f_{H}+d_{H}}$, the $\mathrm{HH}$ type loses its incentives to migrate too quickly for the non trading population to vanish.

Allowing for migration therefore does not affect the basic results, but modifies the transition to an LRE in an interesting way.

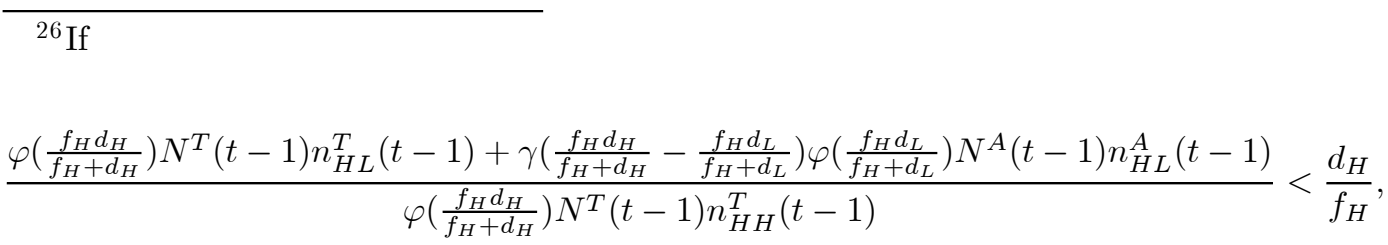

then the reverse occurs. One has $p_{f}=\frac{d_{H}}{f_{H}+d_{H}}, u_{H H}^{T}=u_{H H}^{A}$, and $m_{H H}=0$. The $L$ allele becomes more frequent in the trading population, and disappears more quickly from the autarkic one. 


\section{The impact of trade on collective fitness: the role of environmental shocks}

One question of interest is: does a population which trades do better than one which does not, by enough to drive out the latter from existence in the longrun? As we have seen above, for the same initial distribution of genotypes a trading society will have a larger population in the long-run than a non trading one, but these two populations will grow at the same rate. Hence, the former will be larger than the latter but will not eliminate it. If, on the other hand, it were the case that trade increased population growth, then a trading population would eventually become infinitely large relative to a non trading one, so that the latter has been eliminated in relative terms. ${ }^{27}$

It turns out that trade achieves a greater population growth rate if one allows for shocks to the environment such that the relative survival value of $H$ vs. $L$-alleles can be inverted. A typical example of such an inversion in nature is skin color. Bears in warm climates are dark, while bears in cold climate are white. Thus an allele favouring a white skin would be considered as " $H$ " in a cold climate, but would have to be re-classified as " $L$ " if there is climate change.

If such environmental changes can occur, then, in some sense, the persistence of " $L "$ genes under trade is an asset for the population as a whole. It allows it to diversify its genetic composition so as to better cope with environmental change.

Going back to the simple model of section 2, and assuming a Leontief fitness function, consider a population without trade. We know that in steady state only the strongest type survives, so that $n_{H H}=1$. This population grows at rate $\nu \varphi_{H H}^{A}$.

Now, assume that this population is subject to an environmental shock such that the productivity of $H H$ at activity $d$ is now $d_{L}$ (the L-environment), rather than $d_{H}$ (the H-environment), while that of type $H L$ is now $d_{H}$. Pop-

\footnotetext{
${ }^{27} \mathrm{~A}$ caveat, however, is that if density eventually has a negative impact on fitness, any differences in population growth rates eventually vanish.
} 
ulation now grows at the rate corresponding to a population entirely made of $H L$ individuals, i.e. $\nu \varphi_{H L}^{A}$.

This suggests that on average, when there are shocks to the environment, population will grow at a rate strictly lower than $\nu \varphi_{H H}^{A}$. Now, this reasoning is not quite correct, since when there are repeated environmental shocks, there is no reason why population should entirely be made of $H H$ types. Rather, as long as environmental shocks do not affect the productivity of the $f$-activity, it will be made of both $H H$ and $H L$ types, with the former tending to outnumber the latter in an H-environment, and tending to disappear in an L-environment. In such a case, survival will always be a weighted average of $\varphi_{H L}^{A}$ and $\varphi_{H H}^{A}$, with strictly positive weights, and population growth will be smaller than $\nu \varphi_{H H}^{A}$.

On the other hand, consider a trading economy with a proportion $n_{H H}$ of $H H$ types, such that

$$
\max \left(n_{H H}, 1-n_{H H}\right)<\frac{d_{H}}{d_{H}+f_{H}} .
$$

According to the results at the end of section 5, this distribution is an equilibrium in both the $\mathrm{H}$-environment and the L-environment. In the $\mathrm{H}$ environment, the "weak" type $H L$ achieves the lowest mortality rate by specializing in the $f$ good, while in the L-environment, the weak type is $H H$ and it is the one which specializes in $f$. Therefore, in both states fitness is the same for both types and given by (7). Consequently, population grows at rate $\nu \varphi_{H H}$.

From there one may argue that if populations with different institutions compete with each other for land and natural resources, trading populations will eventually eliminate non trading ones because of their faster population growth rate. This would not be true in the absence of environmental shocks.

\section{$9 \quad$ A generalization}

The preceding results are based on a very simplified representation of sexual reproduction. Can we extend them to a more realistic situation where chro- 
mosomes come by pair and abilities are determined by alleles at more than two loci?

An extension of this paper (Saint-Paul, 2006) considers an arbitrary number of loci, set in groups of $K$ chromosomes.

It is shown that the preceding results can be extended provided the reproduction process satisfies the following properties:

1.Gene conservation: The distribution of genes among offsprings is the same as among parents.

2. Allele independence: The probability distribution of alleles at a given set of loci, among offsprings of the same parents, conditional on alleles at other loci, must be invariant with respect to these other alleles.

3. Mixing: The probability distribution of alleles among offsprings of the same parents, spans all possible recombinations of the parents' alleles, given that one inherits half one's genes from each parent.

4. Monotonicity: An individual A whose genotype differs from individual $\mathrm{B}$ at only one locus, in that it has more good alleles at that locus than B, would, when mating with the same partner (denoted by $\mathrm{C}$ ) as B, produce a distribution of offsprings who have more good alleles at that locus than the offsprings of $\mathrm{B}$ and $\mathrm{C}$, in a first-order stochastic dominance sense.

If these assumptions hold, then the results of Section 5 can be extended. Under autarky, bad alleles disappear in the long run. Under trade, the bad allele only has to disappear at one locus in the long-run. The price system, in a long-run equilibrium, is again pinned down by the strongest genotype's productivity. Finally, for an LRE to hold, genotypes less fit than the fittest should not account for too large a fraction of the population. Proposition 7 in the Appendix shows how condition (6) can be generalized.

\section{Qualifications}

The results derived above are stylized in that they depend on two simplifying ingredients. First, any advantage for one allele over an alternative eventually 
eliminates the latter. Second, specialization makes it possible to eliminate a gentoype's fitness disadvantage due to the presence of an 'inferior' allele at one locus. The results would be milder if one relaxed these assumptions.

If there is an inflow of $L$ alleles due to mutation, these cannot be eliminated in the long run. The result that one gets instead, is that under trade the frequency of $L$ alleles is higher than under autarky. The result that the trading population will achieve a higher overall fitness, and grow faster, than the non trading one, remains.

Trade may not equalize fitness levels between genotypes, if there is a cost (in terms of fitness) to participate in the trade process. The $H H$ type must then, through trade, get a higher fitness than under autarky, to be compensated for this cost. For this, it must be that $p_{d}>\frac{f_{H}}{f_{H}+d_{H}}$, which precludes fitness equalization between $H L$ and $H H$. In such a setting, the $L$ allele would also be eliminated asymptotically. However, (i) it would be at a smaller rate than under autarky; (ii) this rate would go to zero as the cost of trading becomes arbitrarily small, so that (starting from an initial distribution which satisfies (6)) the ratio $n_{H L} / n_{H H}$ at any given future date remains arbitrarily close to its initial value; (iii) if mutation is reintroduced, the $L$ allele no longer disappears and is more frequent under trade, while the conclusion that trading populations achieve higher fitness is unaffected.

Another reason why trade may not eliminate fitness differences, is if gene conservation, and/or allele independence, fail ${ }^{28}$. In such a case, the sexual reproduction process favors some alleles, and trade cannot offset their advantage. If alleles favored by sexual reproduction are also those with lower mortality under autarky, trade can only eliminate part of these allele's fitness advantage. Absent mutation, trade reduces the rate at which weaker alleles are eliminated, but does not prevent their eventual elimination. Under mutation, trade again increases their equilibrium frequency in the population, and the preceding conclusions are unaffected. If sexual reproduction favors

\footnotetext{
${ }^{28}$ As in the case of "linkage disequilibrium", when the presence of an allele at one locus makes it more likely that a given allele at another locus is present in the offspring.
} 
alleles that otherwise have higher mortality, one cannot meaningfully state that these alleles reduce fitness, but again trade will tend to increase their frequency.

\section{Conclusion}

Understanding how genes and culture coevolve is an important step in integrating biology with the social sciences. One important cultural institution of our species is the market. Leaving aside the question of what genetic characteristics of our species led to such a development, this paper has considered the reverse influence: how do markets in turn affect human genetic evolution? The answer it that it makes evolution more selectively neutral by allowing individuals to offset their genetic disadvantages by specializing.

A generalization to small "hunter-gatherer" societies has shown that the lower alleles are eliminated at a lower rate, the larger the group and the more efficient its specialization, while markets can be thought of as a special case when group size is large.

Furthermore, the genetic diversity brought about by markets is beneficial if there are environmental shocks which may reverse the fitness ordering of the alleles; a trading population would then grow faster than a non-trading one. A potential interesting route for further reseach would be to study how these effects could be further enhanced by the use of technology. 


\section{REFERENCES}

Aoki K. (1991) "Time required for gene frequency change in a deterministic model of gene culture coevolution, with special reference to the lactose absorption problem." Theoretical Population Biology, vol. 40, pp. 354-368.

Ashenfelter, Orley, and Allan Krueger (1994), "Estimates of the return to schooling from a new sample of twins", American Economic Review, 84, 5, December

Ashenfelter, Orley, and Cecilia Rouse (1998), "Schooling, Intelligence, and Income in America: Cracks in the Bell curve", Princeton University Industrial Relations Section Working Paper \#407, November

Becker, Gary and Nigel Tomes (1986), "Human Capital and the Rise and Fall of Families", Journal of Labor Economics; 4(3), Part 2 July 1986, pages S1-39.

Boehm, Christopher (1993), "Egalitarian Behavior and Reverse Dominance Hierarchy", Current Anthropology, Vol. 34, No. 3, pp. 227-254 (2001), Hierarchy in the Forest: The Evolution of Egalitarian Behavior.

Bowles, Samuel and Peter Hammerstein (2003), "Does Market Theory Apply to Biology" in Peter Hammerstein, ed., Genetic and Cultural Evolution of Cooperation, MIT Press 2003, pp 153-165

Bouchard, T.J. and M. McGue (1981), "Familial studies of intelligence: A review", Science, 212, 4498, 1055-1059

Boyd, Robert and Peter J. Richerson (1985), Culture and the evolutionary process. Chicago: U. of Chicago press

Carlo M. Cipolla, (1994), Before the Industrial Revolution, Third Edition subtitled "European Society and Economy 1000-1700", Norton.

Cavalli-Sforza, L; and M.W. Feldman, Cultural transmission and evolution; Princeton: Princeton University Press.

Cawley, John, K. Conneely, James Heckman, and Edward Vytlacil (1996), "Measuring the effects of cognitive ability", NBER Working paper 5645, July

Dahlberg, F. (1981), Woman the gatherer, Yale U. Press, New Haven. 
Debreu, Gerard (1959), Theory of Value. Wiley, New York.

Devlin, Bernie, Stephen Fienburg, Daniel Resnick and Kathryn Roeder (1997), Intelligence, Genes, and Success: Scientists Respond to the Bell Curve.

Galor, Oded and Omer Moav (2000), "Natural selection and the origins of economic growth", mimeo, Brown U.

Gintis, Herbert (2004), "The hitchhiker's guide to altruism: Gene-culture coevolution and the internalization of norms", Journal of Theoretical Biology, 220,4 .

Hammerstein, Peter, ed. (2003), Genetic and Cultural Evolution of Cooperation, MIT Press.

Herrnstein, Richard and Charles Murray (1995), The Bell Curve: Intelligence and Class Structure in American Life

Hirshleifer, Jack (1977), "Economics from a biological viewpoint", Journal of Law and Economics, 20, 1, 1-52

Horan, Richard D., Bulte, Erwin, and Jason F. Shogren, (2002) "How trade saved humanity from biological extinction: an economic theory of Neanderthal extinction", mimeo, Michigan State University.

Jochim, Michael A. (1988), "Optimal Foraging and the Division of Labor", American Anthropologist Vol. 90, No. 1, pp. 130-136

Jones, Terry L (1996), "Mortars, Pestles, and Division of Labor in Prehistoric California: A View from Big Sur" American Antiquity Vol. 61, No. 2, pp. $243-264$

Lee, R.B. and I. Devore (1968), Man the hunter, Aldine publishing, Chicago.

Lumsden, C.J. and E.O.Wilson (1981),Genes, Mind, and Culture: The Coevolutionary Process. Cambridge MA: Harvard University Press

Mylius, Sido and Odo Diekmann (1995), "On evolutionary stable life histories, optimization and the need to be specific about density dependence", Oikos, 74, 218-224

Robson, Arthur (2001), "The Biological Basis of Economic Behavior", 
Journal of Economic Literature 39

Saint-Paul, Gilles (2006), "Equilibrium allele distribution in trading populations", mimeo, GREMAQ, Toulouse University.

Seabright, Paul (2004), The company of strangers: A natural history of economic life. Princeton University Press.

Smith, Adam (1994), The Wealth of Nations: An Inquiry into the Nature and Causes, Modern Library.

Tang, Y. P.; Shimizu, E.; Dube, G.; Rampon, C.; Zhuo, M.; Liu, G.; and Tsien, J. Z. (1999). "Genetic enhancement of learning and memory in mice" Nature 401:63-69.

Thompson, P.M., Cannon, T.D., Narr, K.L., van Erp, T.G.M., Poutanen, V.P., Huttunen, M., Lönnqvist, J., Standertskjöld-Nordenstam, C.G., Kaprio, J., Khaledy, M., Dail, R., Zoumalan, C.I., Toga, A.W. (2001). "Genetic Influences on Brain Structure", Nature Neuroscience, 4(12):1253-1258

Tsien, J. Z. (2000). Building a brainier mouse. Scientific American. April issue. Vol. 282, p62-68.

Watanabe, Hitoshi, "Occupational Differentiation and Social Stratification: The Case of Northern Pacific Maritime Food-Gatherers", Current Anthropology, Vol. 24, No. 2 (Apr., 1983), pp. 217-219 


\section{Appendix: Asymptotics under migration}

To prove that migration cannot eliminate the non-trading population, note that in the long run one must have $p_{f t}=\frac{d_{H}}{f_{H}+d_{H}}-\pi_{t}$, where $\pi_{t}<<1$. Then it must be that $m_{H H}=\pi_{t} d_{H} \gamma^{\prime}(0)$ and $m_{H L}=\gamma(\Delta u)-\pi_{t} f_{H} \gamma^{\prime}(\Delta u)$, where $\Delta u=\left(\frac{d_{H}}{f_{H}+d_{H}}-\frac{d_{L}}{f_{H}+d_{L}}\right) f_{H}$ is the utility gain to the $H L$ type of moving to the trading population. Similarly, we have

$$
\begin{aligned}
\varphi\left(u_{H H}^{T}(t)\right) & =\varphi^{\prime}\left(u^{*}\right) \pi_{t} d_{H}+\varphi\left(u^{*}\right) ; \\
\varphi\left(u_{H L}^{T}(t)\right) & =-\varphi^{\prime}\left(u^{*}\right) \pi_{t} f_{H}+\varphi\left(u^{*}\right),
\end{aligned}
$$

where $u^{*}=\frac{f_{H} d_{H}}{f_{H}+d_{H}}$. Finally, it must also be that $n_{H L}^{A}(t)=\mu_{t} n_{H H}^{A}(t)$, where $\mu_{t}<<1$. Substituting these into (13),(14), and (15) we can compute $\pi_{t}$ as a function of $\mu_{t}$ :

$$
\pi_{t}=\frac{\gamma(\Delta u) f_{H}}{\varphi^{\prime}\left(u^{*}\right)\left(f_{H}^{2}+d_{H}^{2}\right) x(t-1)+\varphi\left(u^{*}\right) d_{H}^{2} \gamma^{\prime}(0)} \mu_{t},
$$

where $x(t)=N^{T}(t) n_{H H}^{T}(t) /\left(N^{A}(t) n_{H H}^{A}(t)\right)$. Substituting our first-order approximations into (12) and (14) we get

$$
x(t)=x(t-1)\left(1+\frac{\varphi^{\prime}\left(u^{*}\right) d_{H}}{\varphi\left(u^{*}\right)} \pi_{t}\right)+2 \pi_{t} d_{H} \gamma^{\prime}(0) .
$$

Thus, substituting (17) into (18), we can write

$$
x(t)=x(t-1)\left(1+\frac{a \mu_{t}}{b x(t-1)+c}\right)+\frac{d \mu_{t}}{b x(t-1)+c},
$$

where $a, b, c, d>0$ are constants. Clearly, $x(t)-x(t-1)<k \cdot \mu_{t}$. Therefore, $x(t)$ will remain bounded provided the sum $\sum_{t=T}^{+\infty} \mu_{t}$ is convergent. Since $m_{H L}(t)>>m_{H H}(t)$ and $u_{H L}^{A}<u_{H H}^{A}$, (11) and (12) imply that $n_{H L}(t) / n_{H H}(t)$ converges to zero exponentially. Hence $\sum_{t=T}^{+\infty} \mu_{t}$ is convergent, therefore $x(t)$ is bounded. As a corollary, the autarkic population does not vanish relative to the trading population. 


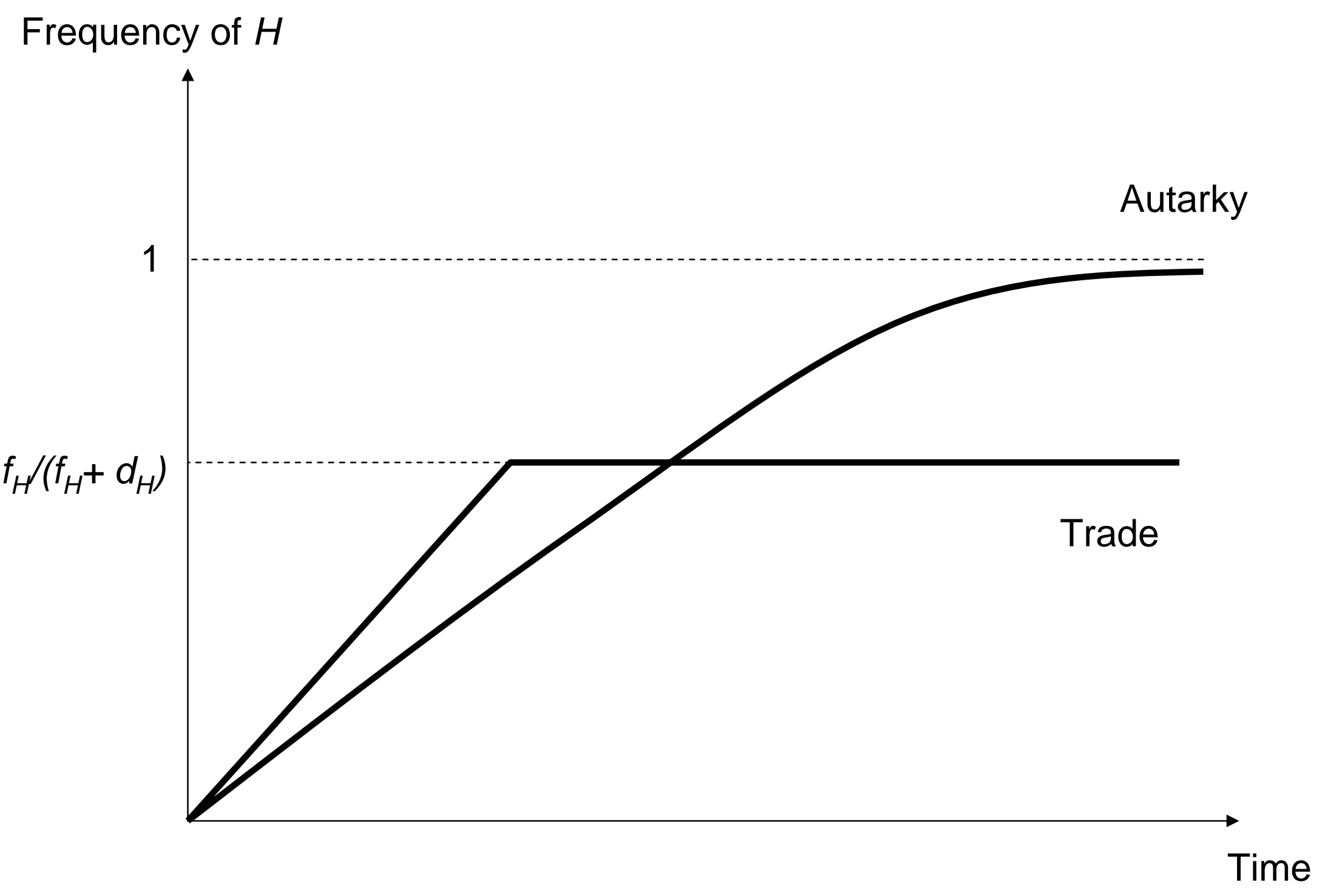

Figure 1: the transition to an LRE 


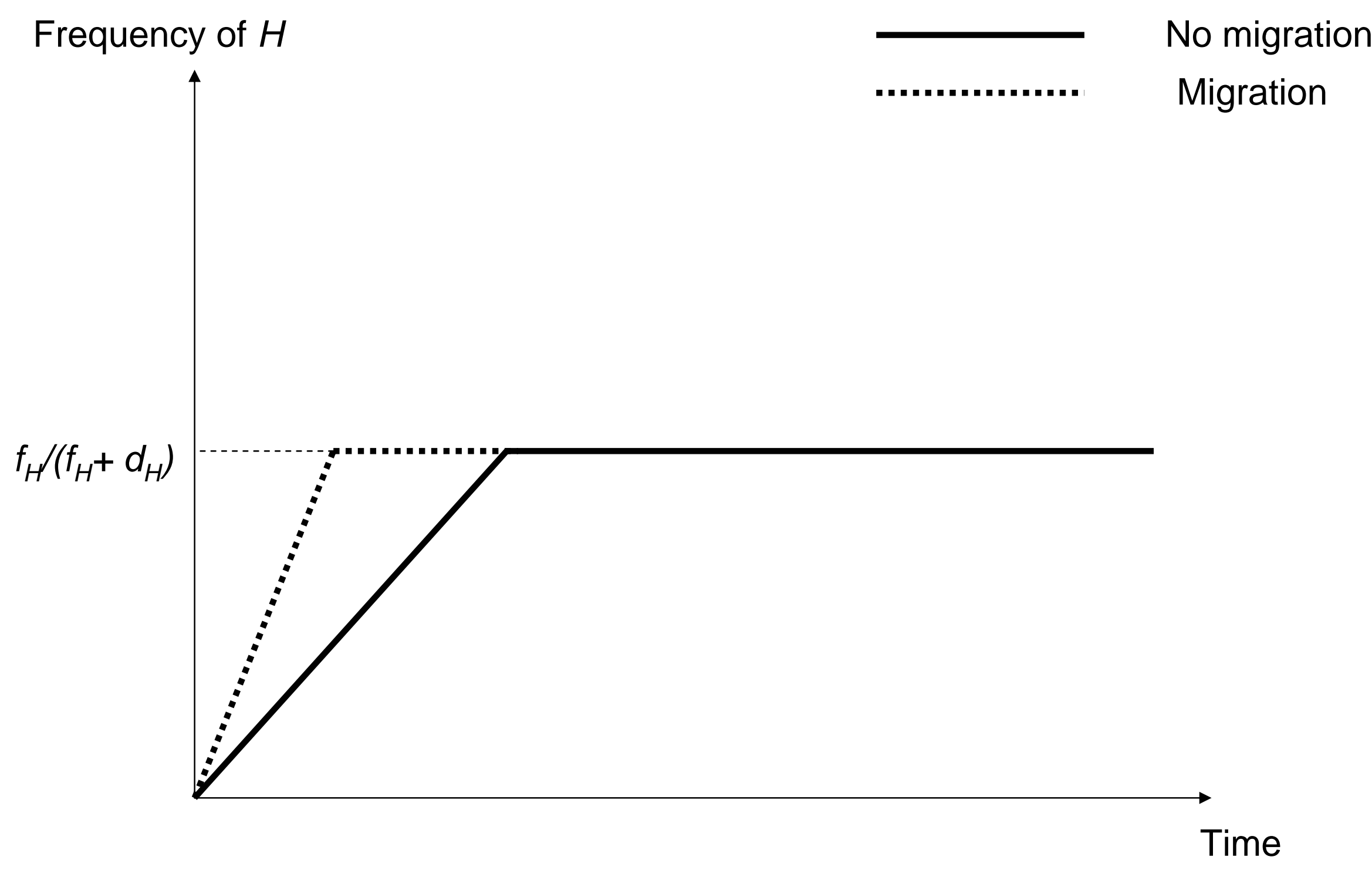

Figure 2: transitional effects of migration on the trading population 


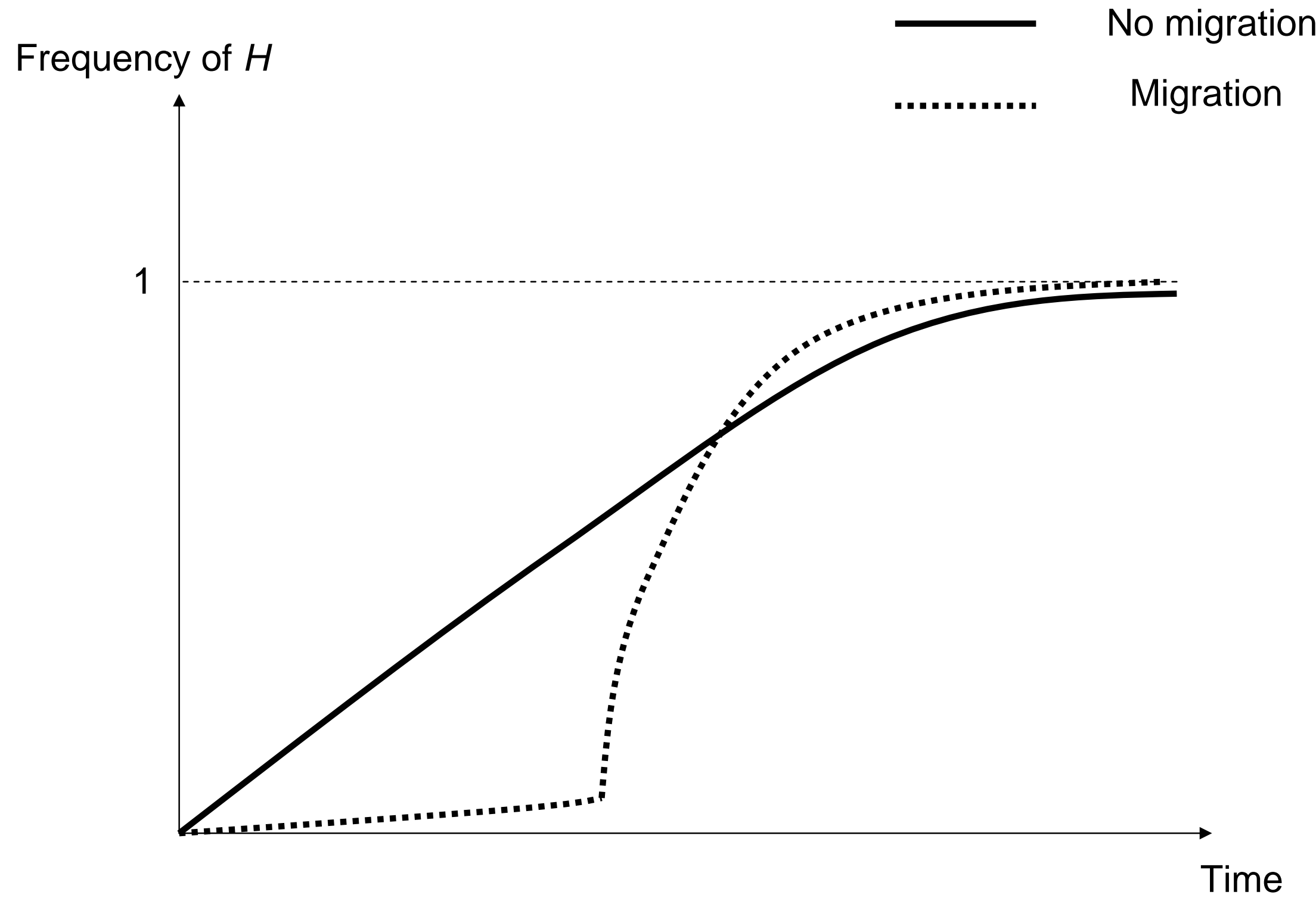

Figure 3: transitional effects of migration on the non trading population 


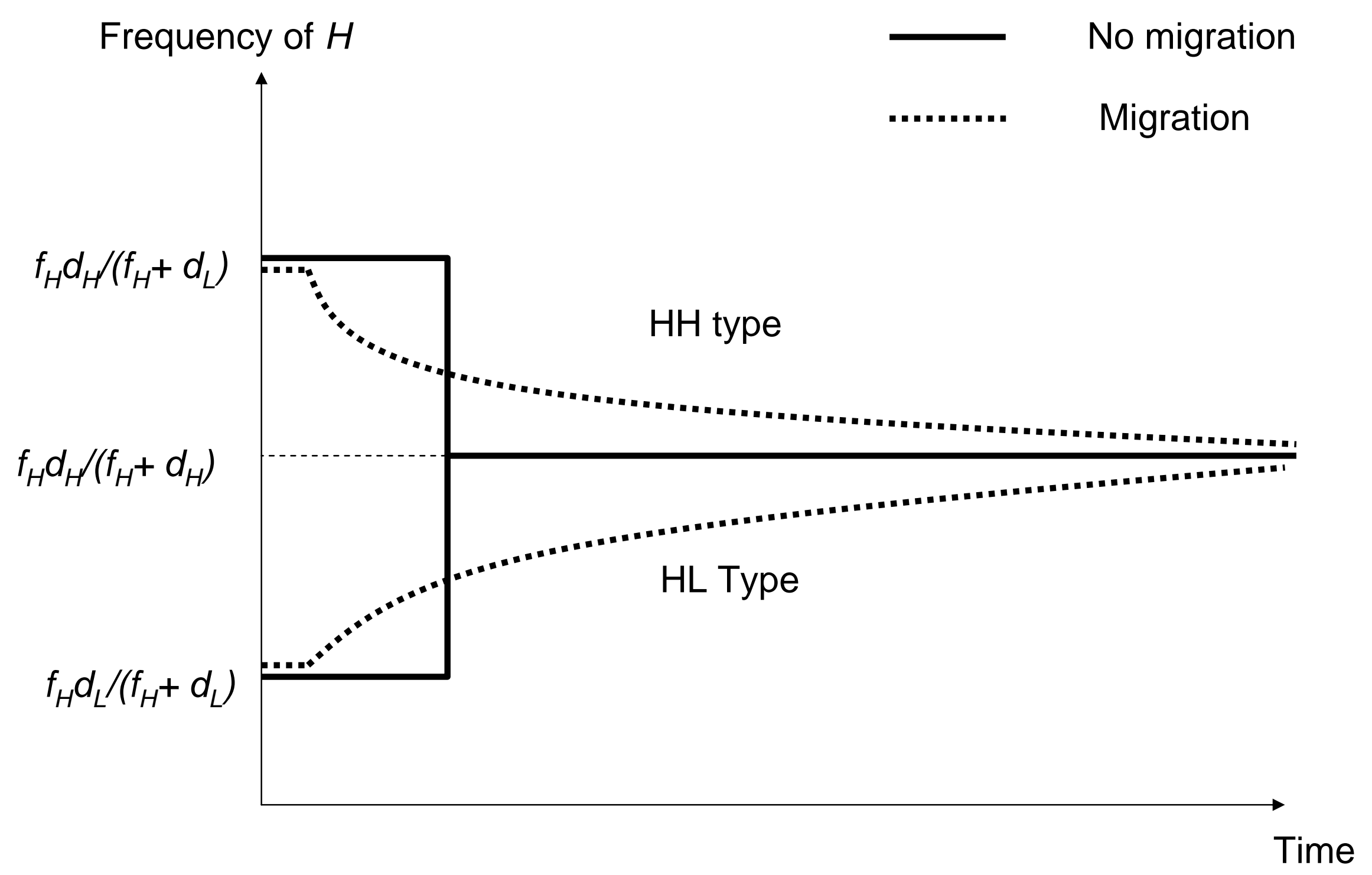

Figure 4: fitness in transition, trading population 